\title{
The Stellar Content in the Central Region of M81
}

\author{
Monique Joly, Catherine Boisson, Didier Pelat \\ Observatoire de Meudon, 92195 Meudon cedex, France
}

\begin{abstract}
Using long slit spectroscopy, we investigate the stellar population and reddening gradients inside the central regions of the nearby spiral galaxy M81. Observational data are analyzed using a new mathematical method. The basic elements to construct the synthetic spectra are taken from a database of star spectra. We show the ability of this method to define detailed stellar populations and to differentiate between age and metallicity effects.
\end{abstract}

\section{Introduction}

The new generation of telescopes allows great improvements in the determination of the stellar content of nearby galaxies since it is now possible to observe individual stars. However, such data are not yet accessible for farther galaxies and other methods, such as the classical stellar population synthesis, have to be used.

In this poster we present results obtained with a new and very powerful mathematical method (Pelat 1997) dedicated to the determination of the best solution (among the many local solutions) in the stellar population synthesis problem.

Using long slit spectroscopy, in the range 5000 - $10000 \AA$, we apply this method to the determination of the stellar population and reddening gradients inside the central 25 arcsec of the nearby spiral galaxy M81 (Serote Roos et al. 1998).

\section{The Method}

A way to derive the stellar population of a galaxy is to fit its spectrum by a combination of stellar spectra extracted from a database. If the data to be fitted are equivalent widths (EW), the combination is a non-linear one:

$$
W_{\text {syn } j}=\frac{\sum_{i=1}^{n_{\star}} W_{j i} I_{j i} k_{i}}{\sum_{i \star 1}^{n_{\star}} I_{j i} k_{i}} .
$$

where $k_{i}$ are the contributions of the stars of type $i$ to the luminosity at a reference wavelength, that satisfy the constraint $\sum_{i=1}^{n_{\star}} k_{i}=1$; and $W_{j i}, I_{j i}$ are the EW and continua of the stars.

The stellar population synthesis is obtained looking for the numbers $k_{i}$ which minimize a distance $D$ defined by: 


$$
D^{2}=\sum_{j=1}^{n_{\lambda}}\left(W_{\text {obs } j}-W_{\text {syn } j}\right)^{2} P_{j}, \quad P_{j} \geq 0 ;
$$

$W_{\text {obs } j}$ are the EW of the absorption lines measured in the galaxy, $W_{\text {syn } j}$ the EW synthesized by means of the stellar database and $P_{j}$ weighting factors related to the $\mathrm{S} / \mathrm{N}$ ratio.

We have a number $n_{\star}$ of stars in the database and a number $n_{\lambda}$ of EW. When $n_{\star} \leq n_{\lambda}$ the problem of finding the 'best' combination of stars that fits the data is fully determined; but it is a complex problem: it is a system of non-linear equations with a key constraint: $k_{i} \geq 0$.

It is probably because of this complexity that the mathematical aspects of the problem did not receive a satisfying treatment; the question of the uniqueness of the solution, for example, was left in uncertainty. Indeed, we have to solve an inverse problem for which many local solutions do exist.

Among these local solutions there generally exists a global solution (the best solution). A new method to search for this solution has been developed.

As a first guess we solve a similar problem, where the system of equations is linearized by taking the weights $P_{j}$ as a function of the synthetic continuum intensity. This problem is only slightly different from the original one but possesses a unique solution and includes the positivity constraint. This solution is very close to the observed galaxy if the $\mathrm{S} / \mathrm{N}$ ratio is reasonable and converges to the true population when $\mathrm{S} / \mathrm{N}$ increases. Then we return to the original problem using that solution as a starting point of a local exploration.

For the particular case of M81 we have $n_{\star}=26$ stars and $n_{\lambda}=40$ lines. The problem is then fully determined and the stellar population synthesis can be computed.

\section{Results}

Table 1 gives the stellar populations (the $k_{i}$ ) obtained for three regions extracted from the 2-D spectrum of M81: the nucleus and two rings. The first column lists the stellar types included in the library. The two last lines display the mathematical distance $D$ (Equn. 2) and the internal reddening, $\mathrm{E}_{B-V}$, determined as the correction to be applied to the observed continuum to match the synthetic one. Figure 1 shows the synthetic spectra (dashed line) superimposed to the observed ones after reddening correction (full line).

The dominant population in the central region of M81 is from an old and evolved population of cool dwarf and giant stars ( $K$ and $M)$, on which is superposed a population of hot massive stars represented by the B3V type. The population is metal rich and almost no dust is detected. The hot component may probably be attributed to a population of extreme horizontal branch stars (EHB) and post asymptotic giant branch stars (PAGB) of high metallicity.

The stellar population in the nucleus has a larger contribution of giants than in the outer regions (rings 1 and 2), where late main sequence stars dominate the radiation. In ring 1 a small contribution from supergiants is present which together with a blue component slightly stronger might indicate the occurrence of some star formation in the recent past $\left(\sim 2 \cdot 10^{7}\right.$ years old). 
Table 1. The stellar populations in the central region of M81: the contributions $k_{i}$ to the luminosity at $\lambda_{0}=5450 \AA$. ( $\mathrm{r}$ is for metal rich stars, $\mathrm{w}$ for metal poor)

\begin{tabular}{|l||c|c|c|}
\hline stars & $\begin{array}{c}\text { nucleus } \\
\mathrm{R}=35 \mathrm{pc} \\
\%\end{array}$ & $\begin{array}{c}\text { ring } 1 \\
\mathrm{D=70} \mathrm{pc} \\
\%\end{array}$ & $\begin{array}{c}\text { ring } 2 \\
\mathrm{D}=160 \mathrm{pc} \\
\%\end{array}$ \\
\hline O5V & 0 & 0 & 0 \\
B3V & 12 & 19 & 13 \\
A13V & 0 & 0 & 0 \\
F2V & 0 & 0 & 0 \\
G4V & 0 & 0 & 0 \\
G9K0V & 0 & 0 & 0 \\
K5V & 0 & 0 & 0 \\
r G0IV & 0 & 0 & 0 \\
r G5IV & 0 & 0 & 0 \\
r K0V & 0 & 0 & 40 \\
r K3V & 33 & 56 & 31 \\
r M1V & 5 & 0 & 0 \\
G7III & 0 & 0 & 0 \\
w K0III & 0 & 0 & 0 \\
K4III & 30 & 0 & 0 \\
M0.5III & 0 & 0 & 12 \\
M4III & 6 & 5 & 4 \\
M5III & 0 & 0 & 0 \\
r G9III & 0 & 0 & 0 \\
r K3III & 0 & 0 & 0 \\
r K5III & 13 & 13 & 0 \\
& & & \\
w K0II & 0 & 0 & 0 \\
w K4II & 0 & 3 & 0 \\
M2I & 0 & 2 & 0 \\
r G2I & 0 & 0 & 0 \\
r K3II & 0 & 1 & 0 \\
\hline$D$ & 25 & 9 & 14 \\
D & 0.0 & 0.03 & 0.05 \\
\hline
\end{tabular}




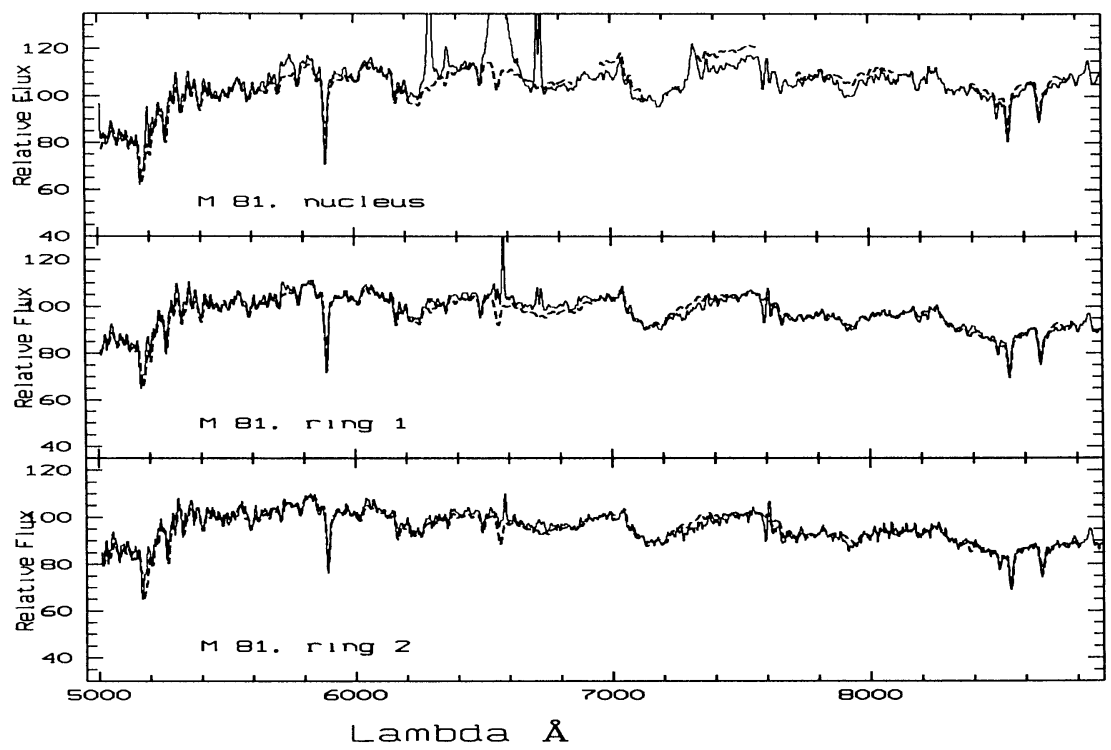

Figure 1. Synthetic spectra (dashed line) superimposed on the observed ones after reddening correction (full line).

\section{Conclusions}

This new population synthesis method allows to determine the best solution accessible with the available data and their inherent $\mathrm{S} / \mathrm{N}$ ratio.

When applied to M81 it exhibits the weak population gradient in the bulge of the galaxy, as well as the small contribution of hot stars.

\section{References}

Pelat, D. 1997, MNRAS, 284, 365

Serote Roos, M., Boisson, C., Joly, M., Pelat, D. 1998, MNRAS, submitted 